\title{
Iphone Apps ForCarcinoma / Melonoma Cancer Early Detections Using ABCDE's Steps Method
}

\author{
Marvin Chandra Wijaya
}

\begin{abstract}
There are many type of skin cancer, such as Basal Cell Carcinoma, Squamous Cell Carcinoma, Melanoma. Todays there are a lot of people affected by skin cancer every years. Skin cancer is dangerous disease. A small rash suddenly appeared on the face and other body parts can potentially be a symptom of skin cancer. By using an Iphone can be detected early symptoms of this disease. By using a camera phone can be done there in the shooting. Therefore, as a form of prevention, we can identify or detect skin cancer with 5 steps ABCDE (Asymmetry, Border, Color, Diameter Evolving). These five steps method can be processed using a software. By using the image processing of each step can be done. Iphone apps use IOS Xcode to build skin cancer detection.
\end{abstract}

Index Terms-Carcinoma, melanoma, early cancer detection, Iphone apps.

\section{INTRODUCTION}

Today, with the depletion of the ozone layer in the atmosphere, the more sunlight is harmful to us, excessive sunlight can bring bad effects on the skin. Ultra violet rays from the sun increases the chances of developing skin cancer.

It is necessary to detect as soon as possible so that treatment can be started earlier and the chance to kill the cancer will increase. Educating yourself against skin cancer symptoms so that you can prepare yourself for the detection of skin cancer symptoms.

In general there are 3 types of skin cancer are:

1) Basal Cell Carcinoma ( BCC ) ( $\mathrm{BCC}$ is a type of skin cancer that is most often the case, below the basal cell layer of the outer shell .

2) Squamous Cell Carcinoma ( SCC ) skin cancer is also the most common is the SCC, which is commonly found in cell squamus . SCC is similar to BCC because it often appears in areas directly exposed to UV light such as the skin, scalp, neck, hands , arms , and legs .

3) Melanoma. Melanoma skin cancers including somewhat rare, but this is the most dangerous . Melanoma appears at the bottom of the epidermis

Symptoms of skin cancer may be different depending on the type of skin cancer that attacks. Symptoms usually detected basal cell carcinoma on the face, ears and scalp. You may notice a lump or a pearl shaped like a candle, or a flat scar skin colored or brown.

Symptoms of squamous cell carcinoma can appear on the face, lips, ears or hands. Squamous cell carcinoma looks like a hard red nodule or lesion on the skin scaly, crusty and flat.

Manuscript received January 30, 2015; revised July 20, 2015.

Marvin Chandra Wijaya is with Computer Engineering Departement, Maranatha Christian University, Bandung, Indonesia (e-mail: marvinchw@gmail.com).
Finally, the signs of melanoma can occur throughout the body, even in places that are not exposed to sunlight. Melanoma can be tangible like a large brown spots with dark spots, moles that change size, color, flavor and may bleed, minor wounds with rough boundary red, white, blue, or blue - black, and black sores on the palms, the soles of the feet , fingers or toes (see Fig. 1).

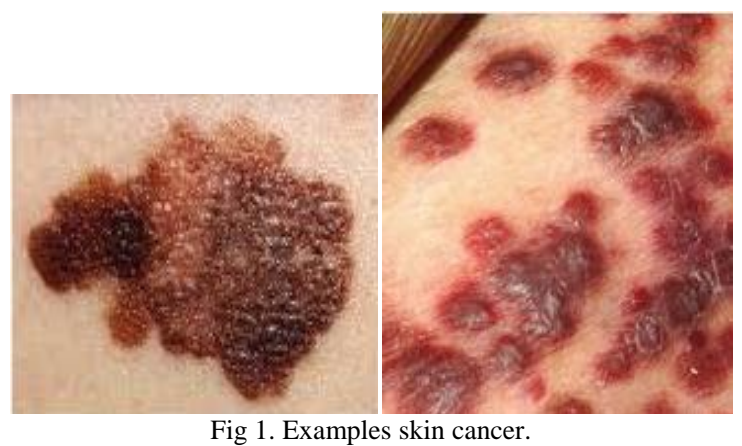

\section{IOS DEVELOPMENT}

To develop iOS apps, need :

1) A Mac computer running OS X 10.8 (Mountain Lion) or later

2) Xcode

3) $\mathrm{iOS}$ SDK

Xcode is Apple's integrated development environment (IDE). Xcode includes a source editor, a graphical user interface editor, and many other features. The iOS SDK extends the Xcode toolset to include the tools, compilers, and frameworks you need specifically for iOS development.

Xcode is an IDE (Integrated Development Environment) used by Mac and iOS developers to build applications. It's not just a code editor: it has a variety of additional goodies baked in like great autocomplete support, static code analysis (it finds bugs in code before compile, including memory leaks) and a variety of debugging and performance tools. We could use TextMate or BBEdit and then use command line tools to do the final compilation, but most developers choose to do it all within Xcode. I use Xcode for all app development.

We can download the latest version of Xcode for free from the App Store on Mac. The iOS SDK is included with Xcode.

Xcode includes several built-in app templates that you can use to develop common styles of iOS apps, such as games, apps with tab-based navigation, and table-view-based apps. Most of these templates have preconfigured interface and source code files for you to start working with

Xcode includes everything you need to create an app. It not only organizes the files that go into creating an app, it provides editors for code and interface elements, allows we to build and run your app, and includes a powerful integrated 
debugger.

\section{A. Interface Builder}

Interface Builder is an application that lets you build your interfaces visually. Built-in objects like buttons, tab bars, sliders and labels can easily be dragged onto your app's interface and then configured by tweaking the palettes and panels. We can also use Interface Builder to connect targets and actions (what happens when an interface element is acted on by a user, and what object handles the action) as well as manipulate controllers and object bindings.

\section{B. Frameworks}

Frameworks is one of the most important for API programming. Without frameworks and APIs developers wouldn't easily be able to create applications that run on Mac OS X or iOS. Apple provides dozens of frameworks that enable developers to do things like create user interfaces, write networking code, encrypt important information, draw graphics to the screen, play audio and video, save data and passwords, take pictures, display webpages and much more.

The frameworks that Apple provides start off with a rich set of commands and tools upon which to build applications. Without the various frameworks that Apple provides, every developer would be reinventing the wheel over and over again. There are lots of goodies given in the various Cocoa frameworks so, many times, an incredibly complex thing can be accomplished in only a few lines of code. An example of this is automatically fetching a file on the Internet by its URL, parsing this file, then stuffing it into a data structure that can manipulate instantly is just a one-liner!.

\section{Objective-C Concepts}

Objective- $\mathrm{C}$ is built on top of the $\mathrm{C}$ programming language and provides object-oriented capabilities and a dynamic runtime. Elements of $\mathrm{C}$ object, such as primitive types (int, float, and so on), structures, functions, pointers, and control flow constructs (while, if...else, and for statements). Also can access to the standard $\mathrm{C}$ library routines, such as those declared in stdlib.h and stdio.h.

\section{Objects Are Building Blocks for Apps}

Objects package data with related behavior. An app is a large ecosystem of interconnected objects that communicate with each other to perform specific tasks, such as displaying a visual interface, responding to user input, and storing information. You use many different types of objects to build your app, ranging from interface elements, such as buttons and labels, to data objects, such as strings and arrays.

\section{E. Classes Are Blueprints for Objects}

A class describes the behavior and properties common to any particular type of object. In the same way that multiple buildings constructed from the same blueprint are identical in structure, every instance of a class shares the same properties and behavior as all other instances of that class. You can write your own classes or use framework classes that have been defined for you.

A fundamental concept in Objective-C programming is class inheritance, the idea that a class inherits behaviors from a parent class. When one class inherits from another, the child - or subclass - inherits all the behavior and properties defined by the parent. The subclass can define its own additional behavior and properties or override the behavior of the parent. Thus you can extend the behaviors of a class without duplicating its existing behavior.

A class is a blueprint that describes the state and behavior of a particular set of objects. It typically models a concept or a thing from the real world, for example, an Animal. AnAnimal class would probably define some variables like the number of legs it has, its height and weight, and also behaviors, like running, eating and sleeping. An object of a given class is called an instance of the class. Each new instance you create can have its own values for the data. You could create 100 instances of the Animal class and set each one's variables to something different. Methods are behaviors a class possesses, and in Objective-C, you can call a method directly on the class itself or on an instance of it. These two types of methods are called class methods and instance methods.

\section{F. Objects Communicate Through Messages}

Objects interact by sending each other messages at runtime. In Objective-C terms, one object sends a message to another object by calling a method on that object.

\section{G. Protocols Define Messaging Contracts}

A protocol defines a set of behaviors that are expected of an object in a given situation. A protocol comes in the form of a programmatic interface, one that any class may implement. Using protocols, two classes distantly related by inheritance can communicate with each other to accomplish a certain goal, such as parsing XML code or copying an object.

Any class that can provide useful behavior to other classes can declare a programmatic interface for vending that behavior anonymously. Any other class can choose to adopt the protocol and implement one or more of the protocol's methods, making use of the behavior.

\section{ABCDE’s STEP SKIN CANCER DETECTION}

There are five steps to detect skin cancer using ABCDE's Step skin cancer early detection. Every letters have different meaning. A is mean Asymmetry, B is mean Border, $\mathrm{C}$ is mean Color, D is mean Diameter and $\mathrm{E}$ is mean Evolving. Explanation for every steps are:

1) Asymmetry. If there are stains that appear on the skin asymmetrical, skewed, and irregular then you should be aware that the stain could be an indicator of the symptoms of skin cancer.

2) Border. If the stain has no boundaries and irregular, it can be a symptom of skin cancer.

3) Color. Stains that appear due to the influence of pigmentation usually have a uniform color. However, if the color of the stain is uneven, it can be a symptom of skin cancer.

4) Diameter. If the stain appears to have a size of 4 to $6 \mathrm{~mm}$, it can be a symptom of skin cancer.

5) Evolving. Note the stains that appear on skin, if the stain is growing and growing, it could be a symptom of skin cancer. Stain on common skin like moles, freckles, and birthmarks relatively constant growth over time. 


\section{DESIGN}

First we have to capture 22 images, using iphone camera or using image file stored in iphone. Then apps calculate length of images. First image pre processing is greyscale image processing. After all images processed, then with certain threshold, image will separated stain from background. After found stain location, then apps will calculate stain diameter. After all images processed, then the data will store in iphone storage.

Flowchart for skin cancer early detections shown in Fig. 2.

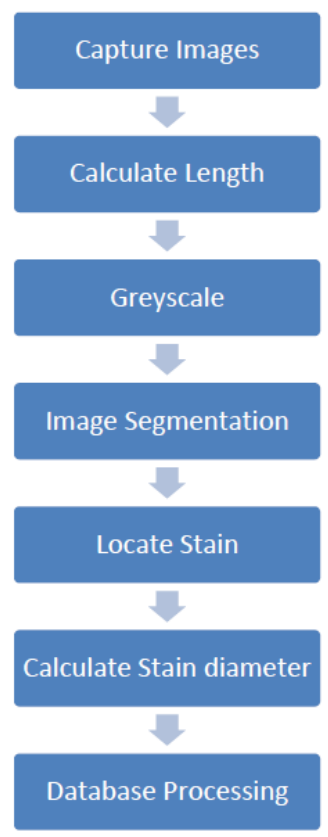

Fig. 2. Flowchart.

\section{A. Greyscale Image Processing}

A common strategy is to use the principles of photometry or, more broadly, colorimetry to match the luminance of the grayscale image to the luminance of the original color image. This also ensures that both images will have the same absolute luminance, as can be measured in its SI units of candelas per square meter, in any given area of the image, given equal whitepoints. In addition, matching luminance provides matching perceptual lightness measures, such as $L^{*}$ which is determined by the luminance $Y$.

To convert a color from a colorspace based on an RGB color model to a grayscale representation of its luminance, weighted sums must be calculated in a linear RGB space, that is, after the gamma compression function has been removed first via gamma expansion [4].

For the sRGB color space, gamma expansion is defined as

$$
C=\left\{\begin{array}{c}
\frac{C_{s r g b}}{12.92} \quad C_{s r g b} \leq 0.04045 \\
\left(\frac{C_{s r g b}+0.055}{1.055}\right)^{2.4} \quad, C_{s r g b}>0.04045
\end{array}\right.
$$

where $C_{\mathrm{srgb}}$ represents any of the three gamma-compressed sRGB primaries ( $R_{\mathrm{srgb}}, G_{\mathrm{srgb}}$, and $B_{\mathrm{srgb}}$, each in range $\left.[0,1]\right)$ and $C_{\text {linear }}$ is the corresponding linear-intensity value $(R, G$, and $B$, also in range $[0,1])$. Then, luminance is calculated as a weighted sum of the three linear-intensity values. The sRGB color space is defined in terms of the CIE 1931 linear luminance $Y$, which is given by

$$
Y=0.2126 R+0.7152 G+0.0722 B
$$

The coefficients represent the measured intensity perception of typical trichromat humans, depending on the primaries being used; in particular, human vision is most sensitive to green and least sensitive to blue. To encode grayscale intensity in linear RGB, each of the three primaries can be set to equal the calculated linear luminance $Y$ (replacing R, G, B by $Y, Y, Y$ to get this linear grayscale). Linear luminance typically needs to be gamma compressed to get back to a conventional non-linear representation. For sRGB, each of its three primaries is then set to the same gamma-compressed $Y_{\text {srgb }}$ given by the inverse of the gamma expansion above as

$$
Y_{s r g b=}\left\{\begin{array}{c}
12.99 Y, \quad Y \leq 0.0031308 \\
1.055 Y^{1 / 2.4}-0.055 \quad Y>0.0031308
\end{array}\right.
$$

Because the three sRGB components are then equal, it is only necessary to store these values once in sRGB-compatible image formats that support a single-channel representation. Web browsers and other software that recognizes sRGB images will typically produce the same rendering for a such a grayscale image as it would for an sRGB image having the same values in all three color channels.

\section{B. Image Segmentation}

This includes segmentation of an image based on thresholding of histogram features and gray level thresholding is perhaps the simplest example of this technique. This is particularly suitable for an image with region or object of uniform brightness placed against a back ground of different gray level. A threshold can be applied to segment the object and background. Mathematically the threshold can be defined as follows.

$$
r_{i}, j=\left\{\begin{array}{cc}
1 & P_{i, j} \geq T \\
0 & P_{i, j}<T
\end{array}\right.
$$

where $r_{i, j}$ is the resulting pixel at co-ordinate $(i, j), p_{i, j}$ is the pixel of input image and $T$ is the value of threshold.

Fig. 3 shows first start windows for cancer early detection apps.

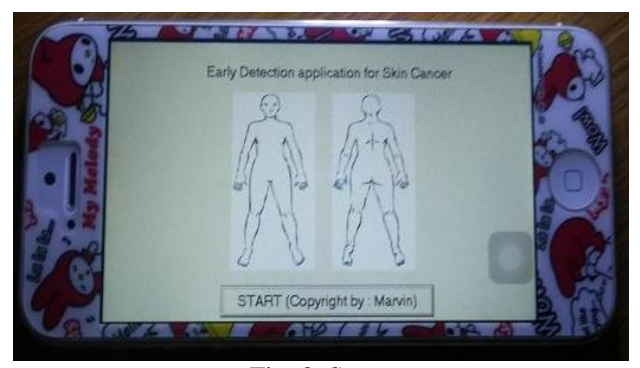

Fig. 3. Start. 
Fig. 4 shows guidance to capture image, there are 22 images of part body can be clicked. For example if user click upperbody capture images, Fig. 4 shown. User can choose using internal camera or using image file.

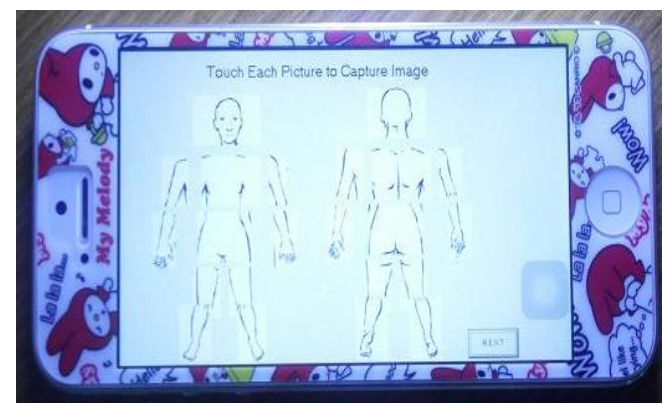

Fig. 4. Guidance for capture image.

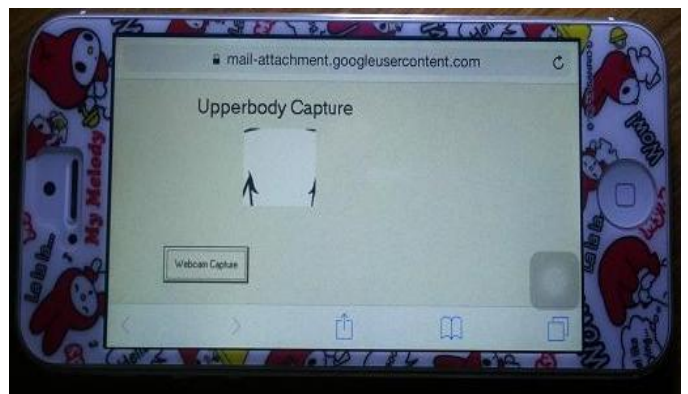

Fig. 5.Upperbody capture.

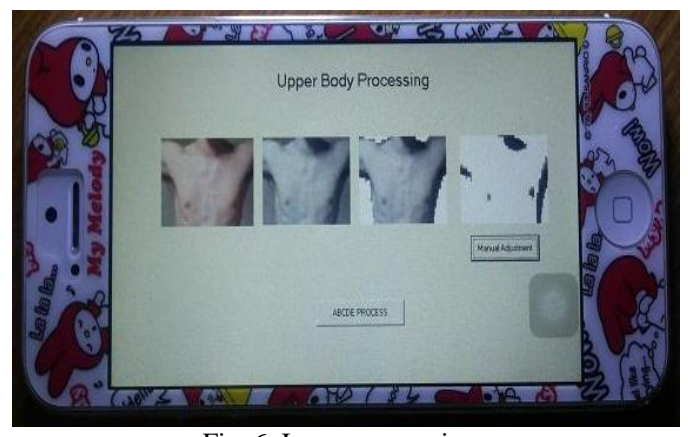

Fig. 6. Image processing.

After all part of body are clicked. There are twenty two (22) part of boy should capture by Iphone Camera or using file in Iphone storage.

Then button $\mathrm{N}$ E X T will appear to continue to next processes. In Fig. 6, there are three image processing :

- greyscale processing,

- image segmentation
- and stain location.

If the results are not as expected, it can be done with manual adjustment. After completion of the next button can be selected.

When user click ABCDE Processing button, Fig. 7 will shown.

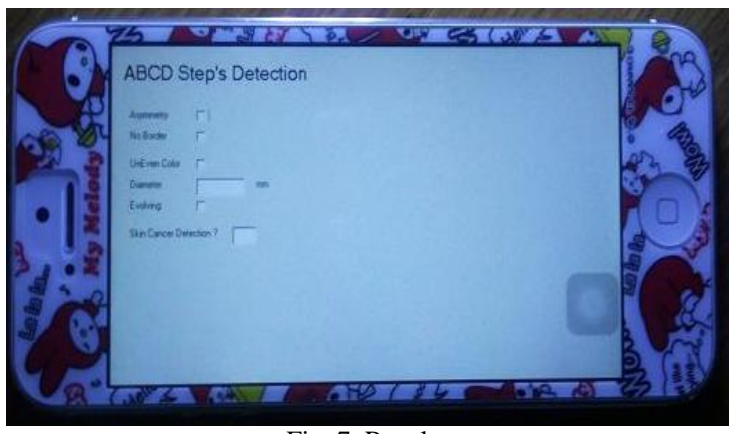

Fig. 7. Result.

\section{CONCLUSION}

The Iphone apps have been going well, but still needs some improvement and refinement.

\section{REFERENCES}

[1] C. Herman, D. Penatalaksanaan, and K. K. Dini, Fkui, 2001.

[2] M. Edi, K. Kulit, and S. S. Bhakti, Ngajuk, 2013

[3] L. Wiliam and C. Linda, The Skin Cancer Answer: The Natural Treatment for Basal and Squamous Cell Carcinomas and Keratoses, 1998

[4] R. L. Jonathan, "A textbook of skin cancer and its mimics," Skin Cancer, p. 909, 2006

[5] Deteksi Kanker Kulit Lewat Tahi Lalat. [Online]. Available: http://www.parenting.co.id/article/dunia.mama/deteksi.kanker.kulit.le wat.tahi.lalat/001/006/643

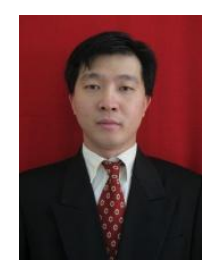

Marvin Chandra Wijaya was born in 1973, in Bandung, Indonesia. Marvin Chandra Wijaya completed his bachelor of electronic in Maranatha Christian University Bandung in 1996. Then, he completed his master of business in Parahyangan Christian Unviersity, Bandung in 1999. Then he completed his master of computer engineering in Institute Teknologi Bandung (ITB), Bandung in 2001

Now, he is a senior lecturer at Computer Engineering Department, Maranatha Christian University, Bandung, Indonesia since 1996. He is also a writer of Computer Books. His research field is in microprocessor and image processing.

Marvin Chandra Wijaya one is member a of American Society for Research. He is a jury of Indonesia Robotic Olympiad. He is a reviewer of Compile Journal and Zenith Journal. 